\title{
Direct Quadrature Conditional Moment Closure for Modelling of Turbulent Combustion
}

\author{
Shaukat Ali • Alexander Vikhansky • Terese Løvås
}

Received: 12 April 2010 / Accepted: 3 December 2010 / Published online: 5 January 2011

(C) The Author(s) 2010. This article is published with open access at Springerlink.com

\begin{abstract}
We present a method of direct quadrature conditional moment closure (DQCMC) for the treatment of realistic turbulence-chemistry interaction in computational fluid dynamics (CFD) software. The method which is based on the direct quadrature method of moments (DQMOM) coupled with the conditional moment closure (CMC) equations is in simplified form and easily implementable in existing CMC formulation for CFD. The observed fluctuations of scalar dissipation around the conditional mean values are captured by the treatment of a set of mixing environments, each with its pre-defined weight. Unlike the early versions of the DQCMC method the resulting equations are similar to that of the first-order CMC, and the "diffusion" term is strictly positive and no correction factors are used. We present results for two mixing environments where the resulting matrices of the DQCMC can be inverted analytically. We have performed this analysis for a simple hydrogen flame using a multi species chemical scheme containing nine species. The effects of the fluctuations around the conditional means are captured accurately and the predicted results are in very good agreement with observed trends from direct numerical simulations. Furthermore, the differences between the first order CMC and DQCMC are discussed.
\end{abstract}

Keywords Turbulent flames $\cdot$ DQCMC $\cdot$ Quadrature method of moments $\cdot$ CMC

This paper was submitted to Flow, Turbulence and Combustion special issue for COCCFEA final workshop papers.

S. Ali · A. Vikhansky · T. Løvås

School of Engineering and Material Sciences,

Queen Mary University of London,

Mile End Road, London, E1 4NS, UK

T. Løvås $(\bowtie)$

Department of Energy and Process Technology,

Norwegian University of Science and Technology,

Kolbjorn Hejes vei 1b, 7491, Trondheim, Norway

e-mail: terese.lovas@ntnu.no 


$\begin{array}{ll}\text { Abbreviations } & \\ \alpha & \text { Environment index } \\ w_{\alpha} & \text { Probability weight of environment } \alpha \\ N_{e} & \text { Number of environments } \\ f_{Y \mathbf{Z}} & \text { Joint probability density function of mixture fraction Y and mass } \\ Y & \text { concentration } \boldsymbol{Z} \\ \mathbf{Z} & \text { Mixture fraction } \\ i & \text { Vector of mass fractions } \\ S_{i} & \text { Species index } \\ N_{\Upsilon \Psi} & \text { Chemical source term for species } i \\ t & \text { Joint scalar dissipation rate conditioned on } \Upsilon=v \text { and } \Psi=\psi \\ f_{Y} & \text { Time variable } \\ f_{\mathbf{Z} \mid Y} & \text { Probability density function of mixture fraction } Y \\ \mathbf{Q}_{\alpha} & \text { Conditional probability density function where } \boldsymbol{Z} \text { is conditioned } \\ N_{S} & \text { on } Y \\ N_{\alpha} & \text { Vector of conditional mean species concentrations conditioned } \\ N_{\alpha}^{\prime} & \text { on } Y \text { for the given environment } \alpha \\ Q_{i} & \text { Number of chemical species } \\ N_{m}\left(=N_{S} N_{e}\right) & \text { Conditional scalar dissipation of } Y=y \text { and } \boldsymbol{Z}=Q_{\alpha} \\ \gamma & \text { Fluctuation of conditional scalar dissipation } \\ h_{\alpha} & \text { Mean conditional concentration of species } i \text { averaged over all } \\ \sigma_{Y} & \text { Turbulent mixing rate in the IEM micro-mixing model } \\ & \text { Fluctuation coefficient } \\ & \text { Scalar dissipation variance }\end{array}$

\section{Introduction}

The accurate modeling of mixing and chemical reaction of scalars in turbulent combustion is a problem of huge interest in various fields of engineering and technology and remains a challenging research area. One of the major research focuses in turbulent combustion is non-premixed and moderately premixed flames [1]. A range of successful models are used and from these models; Conditional Moment Closure (CMC) is one of the most capable and well established techniques for closure of the chemical source term in transport equations for turbulent combustion modelling [2].

Simple 1st order CMC only accounts for mean conditional values of system variables conditioned on the mixture fraction. Hence, it does not capture fluctuations or spread around the conditional mean values. However, simulation of the nonpremixed turbulent combustion flames from direct numerical simulation (DNS) shows the difficulty associated with capturing particularly ignition and extinction phenomena with such simplified models as 1st order CMC [3]. The observed spread around the conditional mean at a given mixture fraction for the scalar dissipation rate is significant and results in a corresponding spread in the temperature, or equivalently the reactedness (defined as temperature increment above the initial value) as illustrated in Fig. 1 from previous studies with DNS of non-premixed ignition [4]. It is clear that the turbulent fluctuation leads to the existence of local 


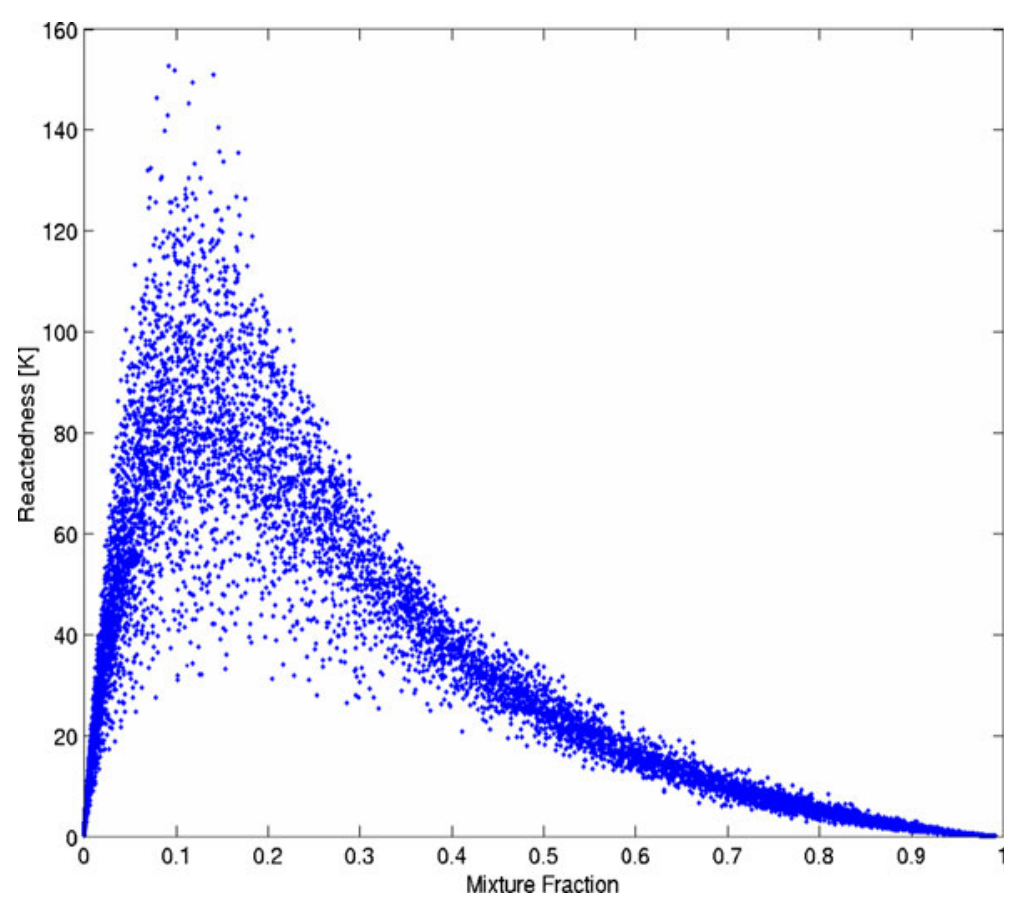

Fig. 1 Reactnedness (temperature increment above initial value) as function of mixture fraction for non-premixed combustion. Scatter plot results from DNS [4]

re-ignition and extinction spots in the flame. Mastorakos et al. [3] showed that the location of highest reactedness is close to stoichiometric conditions and correlated to low scalar dissipation rates. With a significant spread in scalar dissipation rate, it follows that ignition will occur at dissipation rates lower than the averaged mean predicted by 1st order CMC. Therefore, an advanced turbulent combustion model has to account for such fluctuation in the closure equations if ignition and extinction is to be captured.

The significance of fluctuations in the temperature around the conditional mean have been shown in several past studies, e.g. by Kronenburg et al. [5] and Mastorakos and Bilger [6]. Their results show that it is essential to include the temperature fluctuation in the turbulent modelling of CMC for the process involving ignition and extinction of extinction and ignition. Fully burning flames and flames that demonstrate extinction are implicit and broadly used in the practical calculations of turbulent combustion [1]. However, models that can capture both extinction and re-ignition are not easy to formulate since they have to account for the complex interactions between local flame structures (that causes re-ignition) and local fluctuations in turbulent mixing (that causes extinction) [7, 8]. Furthermore, many of such models are formulated to treat isotropic, homogenous turbulence. This is not always the case, and inhomogenous flows will affect an uneven distribution of the fluctuations which is not accounted for in the standard models.

Nonetheless, techniques such as multi mapping conditioning [9] and second order CMC [10] are developed to capture this inconsistency. Both approaches are 
associated with computational and conceptual uncertainties, either by the need for pre-computed probability density functions or in computing second order terms for the unknowns and the corresponding source terms.

The major motivation and aim of the present work is to go beyond the first order CMC model and formulate a computationally efficient method that can account for the non-zero probability that at a given mixture fraction the scalar dissipation rate can take more than one value. The present method is closely related to the direct quadrature method of moments (DQMOM) by Fox and co-workers [11-14]. However, there are some distinct differences which will be outlined below. The present approach has been specifically derived for the CMC-formulation, hence will be referred to as the method of direct quadrature conditional moment closure (DQCMC). The joint probability density function (PDF) is assumed to be a sum of delta functions, all with a given probability weight, $w_{\alpha}$, representing a distinct mixing-environment. The resulting set of transport equations that need to be solved increases with a factor $N_{e}$, where $N_{e}$ is the number of chosen environments. The final conditional mean for each species is the weighed sum of the contributions from each environment.

In this paper we first present in Section 2 the general formulation of the DQCMC method and analyse a special case, namely where $N_{e}=2$, where the resulting matrices of the DQCMC can be inverted analytically. In Section 3 we present the results from employing the derived methodology to a simulation of a mixing and ignition hydrogen flame. Although the chosen examples represent cases with isotropic, homogenous turbulence, the methodology could be extended to inhomogenous flows through a more advanced treatment of the probability weights for the different mixing-environments. Finally, in Section 4 we conclude the work with a short discussion and outline of future work.

\section{DQCMC Methodology}

The underlying objective of the DQCMC is to formulate a model that takes fully into account the fluctuation around the conditional mean in the mixture fraction. The DQCMC method is derived starting from the following transport equation for the spatially homogeneous equation for the joint PDF $[2,11]$ as:

$$
\frac{\partial f_{Y \mathbf{Z}}}{\partial t}=-\frac{\partial S_{i} f_{Y \mathbf{Z}}}{\partial z_{i}}-\frac{\partial^{2} N_{Y Y} f_{Y \mathbf{Z}}}{\partial y^{2}}-2 \frac{\partial^{2} N_{Y Z_{i}} f_{Y \mathbf{Z}}}{\partial y \partial z_{i}}-\frac{\partial^{2} N_{Z_{i} Z_{j}} f_{Y \mathbf{Z}}}{\partial z_{i} \partial z_{j}}
$$

where $\mathbf{Z}=\left(Z_{1}, Z_{2}, \ldots, Z_{\mathrm{N}_{\mathrm{s}}}\right)$ is the mass fractions vector of the reacting species, $N_{\Upsilon \Psi}=\langle D \nabla \Upsilon \nabla \Psi \mid \Upsilon=v, \Psi=\psi\rangle$ is the conditional scalar dissipation rate, and $S_{i}$, is the chemical source term of the $i$ th species.

Integrating Eq. 1 over the reaction-progress space $\mathbf{z}$ yields the transport equation for the PDF of the mixture fraction relating the presumed PDF to the distribution of the scalar dissipation rate:

$$
\frac{\partial f_{Y}}{\partial t}=-\frac{\partial^{2} N_{Y Y} f_{Y}}{\partial y^{2}}
$$

where the calculation of the scalar dissipation rate is performed by double integration of the presumed shape of the PDF of $f_{y}$. As mentioned above, our method is based 
on the direct quadrature method of moments (DQMOM) [11, 12] and is therefore also a presumed composition PDF approach. Furthermore, the conditional PDF, $f_{\mathbf{Z} \mid Y}(y, \mathbf{z})$, is expressed as a multi-peak delta function [11] and hence the joint PDF can be determined by:

$$
f_{Y \mathbf{Z}}(y, \mathbf{z})=f_{Y}(y) f_{\mathbf{Z} \mid Y}(y, \mathbf{z})=f_{Y}(y) \sum_{\alpha=1}^{N_{e}} w_{\alpha} \delta\left(\mathbf{z}-\mathbf{Q}_{\alpha}(y)\right)
$$

$N_{e}$ is the number of environments and $\mathbf{Q}_{\alpha}=\left(Q_{1 \alpha}, Q_{2 \alpha}, \ldots, Q_{\mathrm{N}_{\mathrm{s}} \alpha}\right)$ is the vector of the conditional means of the concentrations of the reacting species in the environment $\alpha$. This implies that for any given value of mixture fraction, the concentrations of a species takes $N_{e}$ selected number of values distributed around the conditional mean and each "realisation", here denoted "environment" to follow the terminology of Fox et al., has its given importance, or weight. The adapted splitting of the joint PDF has the significant numerical advantage of simplifying the derivation of the conditional means for each environment separately.

In the current study we use constant weights $w_{\alpha}\left(\sum w_{\alpha}=1\right)$, while Fox and Raman [11] considered variable weights, $w_{\alpha}(y)$, which evolution is governed by a set of separate differential equations. Smith and Fox studied the variation of the weights in time by comparison with direct numerical simulation (DNS) and found that although the variations are noticeable, and may even shift in terms of which environment is the dominant, the magnitudes are yet around 0.5 [14]. The constant-weights method allows for direct modelling of $N_{s} N_{e}$ moments of the conditional PDF, while the introduction of the variable $w_{e}$ increases this number to $\left(N_{S}+1\right) N_{e}$. In the case $N_{S}>>N_{e}$ the relative gain is modest, i.e., $N_{e} / N_{S}$, while the computational difficulties are significant. When the number of variables is large, this can be handled by the use of tabulation procedures such as ISAT [15]. This approach is chosen by Tang et al. for a multi-species chemical scheme containing 18 species [16]. Such sequential modular procedures are complicated to implement in commercial CFD tools. Hence, we consider the constant-weights method more appropriated for our purposes. Although the simplification of constant weights does not capture the time dependency of the conditional variances, the overall gain from the use of variable weights is small since we are modelling complex systems with number of species greater than one. Furthermore, in the special case of employing two environments with constant weights, the resulting equations can be derived and expressed analytically. This simplifies greatly implementation into CMC tools for CFD.

In order to find an equation for the conditional means of the reacting species in environment $\mathbf{Q}_{\alpha}$, we first substitute Eq. 3 into Eq. 1 to obtain the transport equation for the presumed composition PDF including the multi-peak delta function. To determine how the concentrations are distributed according to the multi-peak delta function PDF we multiply the modified transport equation for the PDF by an arbitrary test function $\phi(\mathbf{z})$ and integrate over $\mathbf{z}$, the composition space. We have that $\int_{-\infty}^{\infty} \delta(x) d x=1$ so we can derive the following useful three relations which will aid the integration of terms III, IV and V in Eq. 1:

$$
\int \phi(\mathbf{z}) N_{Y Y} \delta\left(\mathbf{z}-\mathbf{Q}_{\alpha}\right) d \mathbf{z}=N_{\alpha} \phi\left(\mathbf{Q}_{\alpha}\right),
$$




$$
\begin{aligned}
& \int \phi(\mathbf{z}) \frac{\partial}{\partial z_{i}}\left[N_{Y Z_{i}} \delta\left(z_{i}-\mathrm{Q}_{i \alpha}\right)\right] d \mathbf{z} \\
& =-\int \frac{\partial \phi}{\partial z_{i}} N_{Y Z_{i}} \delta\left(z_{i}-\mathrm{Q}_{i \alpha}\right) d \mathbf{z}=-\left\{N_{\alpha} \frac{\partial Q_{i \alpha}}{\partial y}\right\} \frac{\partial \phi\left(\mathbf{Q}_{\alpha}\right)}{\partial Q_{i \alpha}}, \\
& \int \phi(\mathbf{z}) \frac{\partial^{2}}{\partial z_{i} \partial z_{j}}\left[N_{Z_{i} Z_{j}} \delta\left(z_{i}-\mathrm{Q}_{i \alpha}\right)\right] d \mathbf{z} \\
& =\int \frac{\partial^{2} \phi}{\partial z_{i} \partial z_{j}} N_{Z_{i} Z_{j}} \delta\left(z_{i}-\mathrm{Q}_{i \alpha}\right) d \mathbf{z}=\left\{N_{\alpha} \frac{\partial Q_{i \alpha}}{\partial y} \frac{\partial Q_{j \alpha}}{\partial y}\right\} \frac{\partial^{2} \phi\left(\mathbf{Q}_{\alpha}\right)}{\partial Q_{i \alpha} \partial Q_{j \alpha}},
\end{aligned}
$$

where $N_{\alpha}=\left\langle D|\nabla Y|^{2} \mid Y=y, \mathbf{Z}=\mathbf{Q}_{\alpha}\right\rangle$ is the conditional scalar dissipation rate in the environment $\alpha$. The terms in the figure brackets are not rigorously derived from the joint PDF equation, but postulated in the way similar to the CMC and MECPDF methods [11]. E.g. it is assumed that $N_{Z_{j} Z_{j}}$, which is a conditional expectation of $\left(\nabla Z_{i} \nabla Z_{j}\right)$ can be replaced by conditional expectation of $|\nabla Y|^{2} \partial_{y} Q_{i} \partial_{y} Q_{j}$. This assumption is in line with the main hypothesis of the $\mathrm{CMC}$ that the conditional fluctuations are small. Also, in the above it is assumed that each environment has its own conditional environmental scalar dissipation rate $N_{\alpha}$ as defined above. This has some limitations towards the models ability to account for mixing in z-direction. The assumption implies therefore that not micro mixing between environments are taking place. This limitation will be discussed further below. As we will see bellow, if only one environment is in use, i.e. $N_{e}=1$, the resulting equations are identical to the first-order CMC equations.

The rest of the calculations are straightforward. Corresponding terms in Eq. 1 yield:

$$
\begin{aligned}
& \text { I : } \sum_{\alpha=1}^{\mathrm{N}_{\mathrm{e}}} w_{\alpha} \phi\left(\mathbf{Q}_{\alpha}\right) \frac{\partial f_{Y}}{\partial t}+f_{Y} \sum_{\alpha=1}^{\mathrm{N}_{\mathrm{e}}} w_{\alpha} \frac{\partial \phi}{\partial Q_{i \alpha}} \frac{\partial Q_{i \alpha}}{\partial t}, \\
& \text { II : } f_{Y} \sum_{\alpha=1}^{\mathrm{N}_{\mathrm{e}}} w_{\alpha} \frac{\partial \phi}{\partial Q_{i \alpha}} S_{i}\left(\mathbf{Q}_{\alpha}\right), \\
& \text { III : }-\frac{\partial^{2}}{\partial y^{2}} f_{Y} \sum_{\alpha=1}^{\mathrm{N}_{\mathrm{e}}} w_{\alpha} N_{\alpha} \phi\left(\mathbf{Q}_{\alpha}\right) \\
& \quad=-\frac{\partial}{\partial y}\left[\sum_{\alpha=1}^{\mathrm{N}_{\mathrm{e}}} \frac{\partial N_{\alpha} f_{Y}}{\partial y} w_{\alpha} \phi\left(\mathbf{Q}_{\alpha}\right)+\sum_{\alpha=1}^{\mathrm{N}_{\mathrm{e}}} N_{\alpha} f_{Y} w_{\alpha} \frac{\partial \phi\left(\mathbf{Q}_{\alpha}\right)}{\partial y}\right], \\
& \text { IV : } 2 \frac{\partial}{\partial y}\left[\sum_{\alpha=1}^{\mathrm{N}_{\mathrm{e}}} N_{\alpha} f_{Y} w_{\alpha} \frac{\partial \phi\left(\mathbf{Q}_{\alpha}\right)}{\partial y}\right], \\
& \text { V : }-\sum_{\alpha=1}^{\mathrm{N}_{\mathrm{e}}} N_{\alpha} f_{Y} w_{\alpha} \frac{\partial^{2} \phi\left(\mathbf{Q}_{\alpha}\right)}{\partial Q_{i \alpha} \partial Q_{j \alpha}} \frac{\partial Q_{i \alpha}}{\partial y} \frac{\partial Q_{j \alpha}}{\partial y} .
\end{aligned}
$$


In order to derive Eqs. 9, 10 and 11 we used Eqs. 4, 5 and 6, respectively. Combining Eq. 9 with Eq. 10 one obtains:

$$
\begin{array}{r}
\mathrm{III}+\mathrm{IV}:-\frac{\partial}{\partial y}\left[\sum_{\alpha=1}^{\mathrm{N}_{\mathrm{e}}} \frac{\partial N_{\alpha} f_{Y}}{\partial y} w_{\alpha} \phi\left(\mathbf{Q}_{\alpha}\right)-\sum_{\alpha=1}^{\mathrm{N}_{\mathrm{e}}} N_{\alpha} f_{Y} w_{\alpha} \frac{\partial \phi\left(\mathbf{Q}_{\alpha}\right)}{\partial y}\right] \\
=-\sum_{\alpha=1}^{\mathrm{N}_{\mathrm{e}}} \frac{\partial^{2} N_{\alpha} f_{Y}}{\partial y^{2}} w_{\alpha} \phi\left(\mathbf{Q}_{\alpha}\right)+\sum_{\alpha=1}^{\mathrm{N}_{\mathrm{e}}} N_{\alpha} f_{Y} w_{\alpha} \frac{\partial^{2} \phi\left(\mathbf{Q}_{\alpha}\right)}{\partial y^{2}} .
\end{array}
$$

Calculation of the derivatives in the second term of the above equation by the chain rule and addition of Eq. 11 yields:

$$
\begin{aligned}
\mathrm{III}+\mathrm{IV}+\mathrm{V}:-\sum_{\alpha=1}^{\mathrm{N}_{\mathrm{e}}} \frac{\partial^{2} N_{\alpha} f_{Y}}{\partial y^{2}} w_{\alpha} \phi\left(\mathbf{Q}_{\alpha}\right)+\sum_{\alpha=1}^{\mathrm{N}_{\mathrm{e}}} N_{\alpha} f_{Y} w_{\alpha} \frac{\partial^{2} Q_{i \alpha}}{\partial y^{2}} \frac{\partial \phi}{\partial Q_{i \alpha}} \\
=-\frac{\partial^{2} N f_{Y_{y}}}{\partial y^{2}} \sum_{\alpha=1}^{\mathrm{N}_{\mathrm{e}}} w_{\alpha} \phi\left(\mathbf{Q}_{\alpha}\right)-\sum_{\alpha=1}^{\mathrm{N}_{\mathrm{e}}} \frac{\partial^{2} N_{\alpha}^{\prime} f_{Y}}{\partial y^{2}} w_{\alpha} \phi\left(\mathbf{Q}_{\alpha}\right) \\
+\sum_{\alpha=1}^{\mathrm{N}_{\mathrm{e}}} N_{\alpha} f_{Y} w_{\alpha} \frac{\partial^{2} Q_{i \alpha}}{\partial y^{2}} \frac{\partial \phi}{\partial Q_{i \alpha}},
\end{aligned}
$$

where $N_{\alpha}^{\prime}=N_{\alpha}-N$ is accounting for the fluctuations of the conditional scalar dissipation rate. This parameter, as will be discussed in the following, is a user-set variable in the model. Collecting terms I-V together and making use in Eq. 2 we obtain after some algebra:

$$
\sum_{\alpha=1}^{N_{\mathrm{e}}} w_{\alpha} \frac{\partial \phi}{\partial Q_{i \alpha}}\left(\frac{\partial Q_{i \alpha}}{\partial t}-N_{\alpha} \frac{\partial^{2} Q_{i \alpha}}{\partial y^{2}}-S_{i}\left(\mathbf{Q}_{\alpha}\right)\right)=-\frac{1}{f_{Y}} \sum_{\alpha=1}^{N_{\mathrm{e}}} \frac{\partial^{2} N_{\alpha}^{\prime} f_{Y}}{\partial y^{2}} w_{\alpha} \phi\left(\mathbf{Q}_{\alpha}\right)
$$

It should be noted that the terms collected between the brackets on the left hand side are the classic terms of the 1st order CMC equations. Hence, Eq. 14 shows the contributions for each mixing environment on the right hand side that are not accounted for in the classic formulation.

Equation 14 can for clarity be rearranged such that the governing equation for each species if $\mathbf{Q}_{\alpha}$ is expressed by:

$$
\frac{\partial Q_{i \alpha}}{\partial t}-N_{\alpha} \frac{\partial^{2} Q_{i \alpha}}{\partial y^{2}}-S_{i}\left(\mathbf{Q}_{\alpha}\right)=a_{i \alpha}
$$

where $a_{i \alpha}$ is a solution of the following linear algebraic equation:

$$
\sum_{\alpha=1, i=1}^{\mathrm{N}_{\mathrm{e}}, \mathrm{N}_{\mathrm{s}}} w_{\alpha} \frac{\partial \phi\left(\mathbf{Q}_{\alpha}\right)}{\partial Q_{i \alpha}} a_{i \alpha}=-\frac{1}{f_{Y}} \sum_{\alpha=1}^{\mathrm{N}_{\mathrm{e}}} \frac{\partial^{2} N_{\alpha}^{\prime} f_{Y}}{\partial y^{2}} w_{\alpha} \phi\left(\mathbf{Q}_{\alpha}\right)
$$

The right hand side of Eq. 16 has a clear physical interpretation which includes the local rate of change of mixture fraction PDF, $f_{Y}$, due to the fluctuations in scalar 
dissipation for the given environment $\alpha$ This will in turn affect the final conditional mean values derived from the left hand side. Equations 15 and 16 can be satisfied only for a finite set of functions $\phi$. In the present work we demand that Eqs. 15 and 16 conserve the conditional means (i.e. $\left\langle Q_{i} \mid y\right\rangle=\sum_{t} w_{\alpha} Q_{i \alpha}$ ) and a number of second moments.

Let us introduce the new index $p=(\alpha-1) N_{s}+i$. Note that $p=1, \ldots N_{m}$, where $N_{m}=N_{s} N_{e}$ is the number of the conditional moments which can be correctly reproduced by the distribution (Eq. 3). Then for the set of $N_{m}$ test functions $\phi_{p}(\mathrm{Q})$ Eq. 16 reads:

$$
\sum_{q=1}^{\mathrm{N}_{\mathrm{m}}} A_{p q} a_{q}=b_{p}
$$

where

$$
A_{p q}=w_{q} \frac{\partial \phi_{p}\left(\mathbf{Q}_{q}\right)}{\partial Q_{q}}
$$

and

$$
b_{p}=-\frac{1}{f_{Y}} \sum_{\alpha=1}^{\mathrm{N}_{\mathrm{e}}} \frac{\partial^{2} N_{\alpha}^{\prime} f_{Y}}{\partial y^{2}} w_{\alpha} \phi_{p}\left(\mathbf{Q}_{\alpha}\right)
$$

Equations 14, 15, 16, 17, 18 and 19 are similar to the equations obtained by Fox and Raman [11]. However, they noted that their version of Eq. 15 do not guarantee conservation of the unconditional mean $\left\langle Q_{i}\right\rangle=\sum w_{a} \int Q_{i a}(y) f_{Y}(y) d y$ in the absence of the chemical reactions. In order to ensure mass conservation an additional term has been added ad hoc to the equation. In order to derive the correction term Fox and Raman demanded that summation of Eq. 15 over $\alpha$ should reproduce the 1st order CMC equations [11]. Since the CMC equations are conservative, the resulting model conserves the unconditional means. The equations used in the present study are different. Note that if we substitute $Q_{i}$ as the test functions into Eq. 14, the first $\mathrm{N}_{s}$ equations for the first moments are independent from the higher-order equations. Performing the steps (7-14) in the reverse direction we can show that the system of equations

$$
\sum_{\alpha=1}^{N_{\mathrm{e}}} w_{\alpha}\left(\frac{\partial Q_{i \alpha}}{\partial t}-N_{\alpha} \frac{\partial^{2} Q_{i \alpha}}{\partial y^{2}}-S_{i}\left(\mathbf{Q}_{\alpha}\right)\right)=-\frac{1}{f_{Y}} \sum_{\alpha=1}^{\mathrm{N}_{\mathrm{e}}} \frac{\partial^{2} N_{\alpha}^{\prime} f_{Y}}{\partial y^{2}} w_{\alpha} Q_{i \alpha}
$$

do conserve the mass of the reactants in the absence of the chemical reactions. On the other hand summation of Eq. 15 over does not lead to the 1st order CMC equations as it has been demanded in reference [11]. Note that the 1st order CMC method is based on the assumption that the conditional fluctuations are small, while our task is to loosen these restrictions. For that reason we do not consider the discrepancy between the DQCMC method and 1st order CMC as a drawback. Our model is therefore free of this assumption and can be reduced to 1st order CMC only if the conditional fluctuations are small.

Equation 15 has the clear drawback, that there is no mixing between the environments included. However, mixing between the environments, in direction normal to 
the constant mixture fraction layers, is indeed present and need to be included into the model. This was by Fox and Raman [11] motivated by discrepancies between model and DNS data. In order to overcome this difficulty we follow Fox and Raman [11] and postulate an additional mixing term in the RHS of Eq. 15, which then gives us the final set of transport equations that needs to be solved for the DQCMC:

$$
\frac{\partial Q_{i \alpha}}{\partial t}-N_{\alpha} \frac{\partial^{2} Q_{i \alpha}}{\partial y^{2}}-S_{i}\left(\mathbf{Q}_{\alpha}\right)=\gamma\left(\left\langle Q_{i} \mid y\right\rangle-Q_{i \alpha}\right)+a_{i \alpha}
$$

$\gamma$ is the mixing rate in the IEM micro-mixing model [12] and the first term on the RHS of the DQCMC equation, Eq. 21, thus represents the mixing between the environments. The second term on the RHS is the solution of the system of linear equations resulting from contributions from the chosen moments. This equation has resemblance to both the one-dimensional unsteady flamelet equation [1] and the CMC equations [2], apart from the terms on the RHS. In the CMC these analytically derived terms are represented by the unclosed terms for conditional velocity and conditional turbulent flux which both requires modelling. The unsteady flamelet equations can be interactively coupled to a computational fluid dynamics solver in order to account for fluctuations by updating the flamelet for the local scalar dissipation and its variance calculated from the CFD, the so called representative interactive flamelet (RIF) model [1]. However, as this is performed on each CFD cell, this probably the most computationally expensive approach of all the considered models.

The micro-mixing model used in the present expression will be discussed in more detail below. However, here it can be mentioned that other possible methods to model the mixing between the environments include tabulation of pre-computed premixed flame data [17]. It can be assumed that insignificant mixing occurs between the environments in direction of the mixture fraction. Hence, a simple expression for the micro mixing in terms of interaction with the mean (IEM) is well justified.

In the special case of employing two mixing environments with equal weights $\left(w_{1}=w_{2}=1 / 2\right)$, Eq. 17 has an analytical solution. The set of test functions is chosen as follows: it is assumed that there is one leading variable, e.g., temperature. Then the test functions are $Q_{1}$ (to ensure the conservation of the means) and $Q_{1} Q_{i}$, i.e. the dispersion of the temperature and the correlations between the temperature and the concentration of $i t h$ species. The analytical expression of $a_{i \alpha}$ is in fact found to be independent of the choice of leading variable and will, following the arguments above, take the form:

$$
a_{i \alpha}=-\frac{1}{2 f_{y}} \frac{\partial^{2} N_{\alpha}^{\prime} f_{Y}}{\partial y^{2}}\left(Q_{i 1}-Q_{i 2}\right)
$$

The method postulates the values of the micro-mixing rate $\gamma$ and the spread in the scalar dissipation rate $N_{\alpha}^{\prime}=N_{\alpha}-N$. In the present work the mixing rate is found employing the model of Fox and Raman [11], whereas the initial spread in scalar dissipations is given by $N_{\alpha}^{\prime}=h_{\alpha} \cdot N$ where $h_{\alpha}$ is a user-set fluctuation coefficient. However, if the methodology is coupled with CFD, the estimated fluctuation coefficient can be obtained from the turbulent flow calculation. The conditional mean for each variable is thereby determined by summing up the weighted contribution from each mixing environment: $\left\langle Q_{i} \mid y\right\rangle=\sum w_{\alpha} Q_{i \alpha}$. 
The numerical implementation of the model will then consist of the following steps; firstly the presumed shape of the mixture fraction PDF is defined, most commonly based on a $\beta$-PDF approach as will be discussed below. From this the scalar dissipation is calculated according to the double integration of Eq. 2. The scalar dissipation of each environment, $N_{a}$, is subsequently defined according to the fluctuation coefficient as described above, resulting in $N_{e} N_{s}$ number of ODE's of the form given by Eq. 21 that needs to be simultaneously solved. Note that the extension to 1 st order CMC given by Eq. 22 is analytical and can easily be implemented numerically.

If the initial spread around the conditional means are small, the set of ODE's will not require much additional computational resources as 1st order CMC as the convergence is achieved fast due to similar solutions for each environment. On the other hand, if the initial spread is assumed to be large a greater computational time is expected. However, as will be discussed below the present method is already restricted to conditions with moderate fluctuations around the conditional means due to the simplification that two environments with equal weights are employed in order to obtain an analytical solution. Initial conditions for the fuel and oxidant are given according to the specific flame problem at hand, and the time evolution of Eq. 21 for each species is solved using a numerical ODE solver such as VODPK [17] or LSODE [18].

\section{Results and Discussion}

The analysis is performed for two cases of hydrogen flames; firstly we discuss a case of simultaneous mixing and ignition of a hydrogen flame, and thereafter we show results from a well mixed igniting flame. In the former case the reactants are initially partially non-mixed, gradually mixing to a premixed flame. This is accounted for by assigning the presumed $\beta$-PDF for the mixture fraction with an initially high variance, which gradually is decreasing throughout the simulation. The corresponding mixture fraction pdf is plotted in Fig. 2a at three different instants of time during the simulations and will be discussed in more detail below. Recall that the employed scalar dissipation rate is calculated from the double integration of the presumed shape of the mixture fraction PDF. The initial temperature for the oxidizer which in this case is air is $1100 \mathrm{~K}$ and the initial fuel temperature is $300 \mathrm{~K}$, at one bar of pressure. The chemical mechanism employed in the present work is the $\mathrm{H}_{2}-\mathrm{O}_{2}$ mechanism by Warnatz et al. [19]. It consists of 9 species including nitrogen interacting in 19 reversible reactions.

In the special case of employing two mixing environments with equal weights $\left(w_{1}=w_{2}=1 / 2\right)$ the additional fixed parameters are the constants related to the mixing rate $\gamma$ in Eq. 21 and the presumed spread in scalar dissipation rate $N^{\prime}$ in Eq. 22. For the mixing rate between environments the following definition is employed [12]:

$$
\gamma=C_{Y} \frac{\int f_{Y}(y) N_{Y Y}(y) d y}{\sigma_{Y}^{2}}
$$

where we have set $C_{Y}=2$ according to Smith and Fox [14] as discussed below. The other variables are all obtained from the simulation (the mixture fraction $\operatorname{PDF} f_{y}$; the 
Fig. 2 The presumed PDF (a) and corresponding conditional scalar dissipation rate (b) as function of mixture fraction, at mean mixture fraction of 0.5

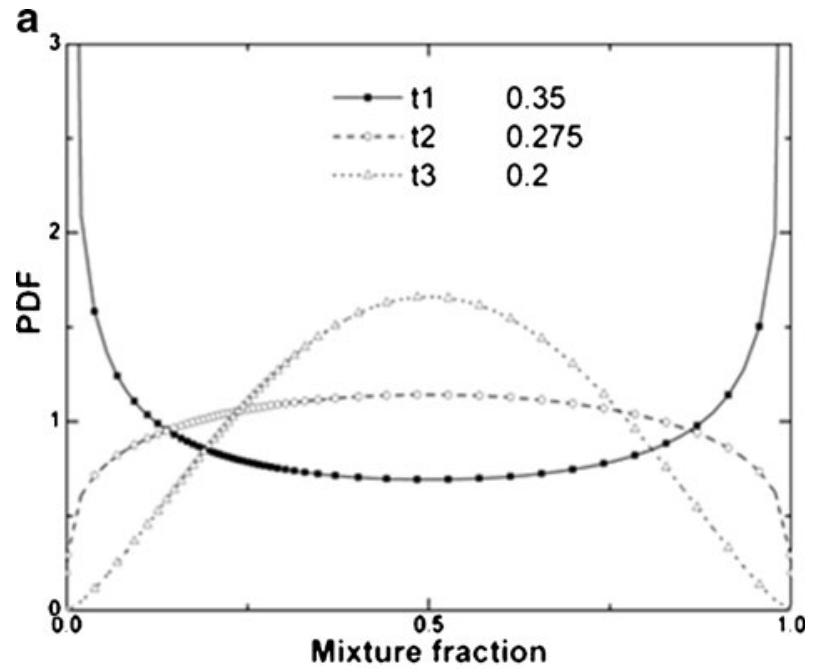

b

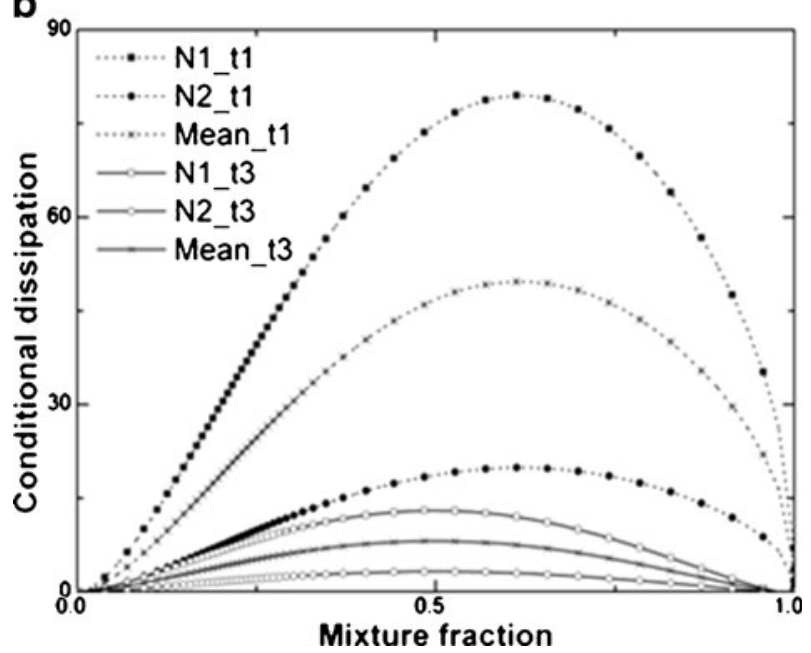

scalar dissipation rate $N_{Y Y}(y)$; and the scalar dissipation rate variance $\sigma$ ). Smith and Fox [14] investigated the validity of the mixing constant $C_{Y}$ in the IEM micro-mixing model. They found that the mixing constant was indeed not constant, but strongly dependent on the Damkohler (Da) number and a time-dependent variable such as the scalar variance. It is clear that a detailed description of the micro-mixing should account for this variation in the mixing constant. However, as concluded by Smith and Fox [14] for low Da numbers, the mixing constant rapidly takes a value around $C_{Y} \approx 2$. Furthermore, Smith and Fox [14] showed that range of contribution of interenvironmental mixing was accurate compared to DNS data for low Da numbers, whereas for increase Da numbers a clear deviation from DNS was observed. For the purpose of developing a simplified and analytical scheme for DQCMC, we adopt this value with the emphasis that the present method is restricted to situations of low Da. It can also be mentioned that other constant values of $C_{Y}$ close to 2 has been tested 
with very little influence on the final result $\left(C_{Y}=1\right.$ gave a temperature difference of around $8-10 \mathrm{~K}$ throughout the flame). In future applications of the present method, a simple time dependency can be employed to account for the time variations observed from DNS.

The constant $h_{a}$ determining the scalar dissipation rate fluctuations for the environments, we have set $h_{1}=1.6$ for the first environment and $h_{2}=0.4$ for the second environment to ensure a significant spread. This is in accordance with the findings of Smith and Fox [14] where DNS was used to evaluate the values of the fluctuation coefficient. In their case of 2 environments and a low Da number, the high and low value of $h_{\alpha}$ was around 1.5 and 0.5 respectively throughout the flame. According to DNS the values varied little across mixture fraction, however with a significant dip in the high valued $h_{1}$ very close to mixture fraction 0 and 1 . Since this is the region of low scalar dissipation rate, this particular structure will not be significant in the modelling. Hence, the fluctuation coefficients are here for simplicity considered to be constant throughout the simulation.

We are showing two different types of simulation results. The first set of results (Figs. 2, 3 and 4) shows an igniting mixture starting from partially mixed conditions with a $\beta$-pdf variance of 0.35 to well mixed mixture with a variance of 0.2 . The second set of results is from a flame with close to stoichiometric conditions and a mean mixture fraction of 0.2. Results are shown for three different times. The time $\mathrm{t} 1$ is the initial time, $\mathrm{t} 2$ is the central time and $\mathrm{t} 3$ is the final time for a fully developed stationary flame. N1 and N2 indicate the results from the two different chosen environments of the analysis and the mean is the average of the weighted two environments.

Figure 2a shows the evolution of the presumed $\beta$-PDF for the different times of the analysis. It is shown that the pdf developed from a partially mixed state (time $\mathrm{t} 1$ ) with a variance of 0.35 to a well mixed condition at time $t 3$ with a narrow variance of 0.2. The presumed PDF is shown to initially have a double peaked shape developing into a PDF with maximum value at a mixture fraction of 0.5 . This corresponds to a fuel rich hydrogen flame, for which the corresponding stoichiometric value is 0.2. This satisfies the experimental result as shown in Fig. 3 in Ref. [2]. Figure $2 b$ shows the corresponding conditional scalar dissipation rate at initial and final times resulting from the two environments with $h_{1}=1.6$ and $h_{2}=0.4$ respectively. The scalar dissipation rates are derived from Eq. 2. in combination with the definition of $N_{\alpha}$ following Eq. 4. for the same time instants. The resulting scalar dissipation is found to be slightly shifted to higher mixture fractions compared to the standard presumed bell-shaped dissipation rate. Since the scalar dissipation is derived from the mixture fraction PDF rather than being presumed, note also that this confirms the correlation between the higher scalar dissipation rate as in environment 1 and a faster mixing resulting in a faster decrease in the scalar dissipation. It can also be noticed that at time $\mathrm{t} 1$ the conditional scalar dissipation is not fully developed as the $\beta$-pdf is not developed (fuel and air is partially mixed). However, at time $\mathrm{t} 3$ as the $\beta$-pdf is developed fully (fuel and air is well mixed), the conditional dissipation takes the well known bell-shape in the mixture fraction space.

Figure 3 a shows the evolution of the conditional temperature resulting from the DQCMC method in comparison with classic first order CMC. It reveals the discrepancy between 1st order conditional mean and the mean from the two environments of the DQCMC scheme. It should be noted that the predicted temperatures of 
Fig. 3 Conditional temperature as function of mixture fraction (a), enlarged section of the conditional temperature following ignition as function of mixture fraction (b) and maximum temperature as function of time (c), at mean mixture fraction of 0.5

Fig. $4 \mathrm{H}$ (a) and $\mathrm{OH}(\mathbf{b})$ mass fractions as function of mixture fraction at conditions with mean mixture fraction of 0.5
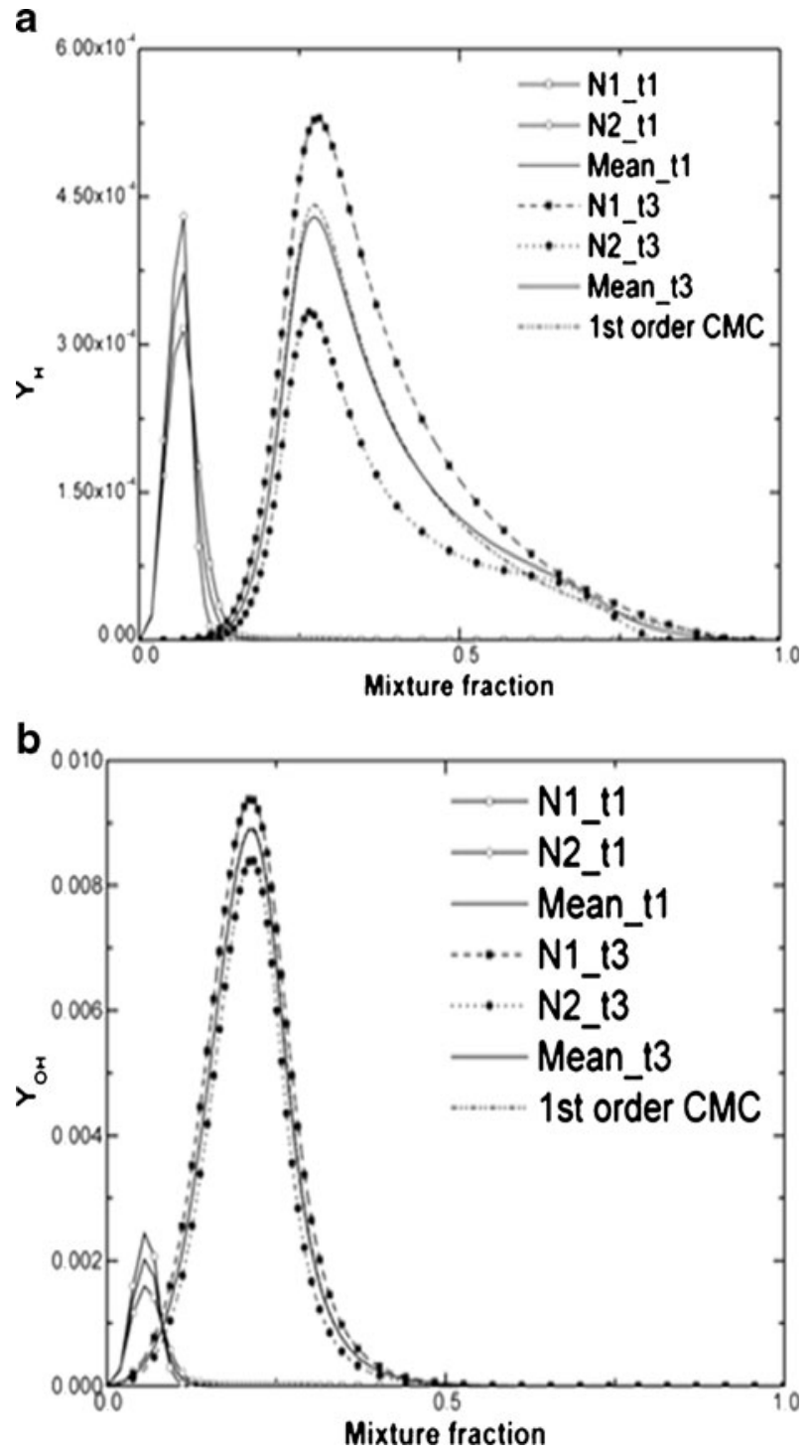

environment 2 is higher, corresponding to the lower scalar dissipation rate in this environment. Figure $3 \mathrm{~b}$ shows an enlargement of the ignition zone of the two environments from the employed technique. It clearly predicts that environment 2 ignites more rapidly than environment 1 . This prediction is in very good agreement with the previous DNS results [3, 4] which shows that there is an inverse correlation between the local temperature and scalar dissipation rate. As discussed by Mastorakos et al. [3] this results in high temperature regions which are located where the scalar dissipation is locally low leading to that the locations of hot-spots (ignition spots) coincides with low scalar dissipation. As shown in Fig. $3 b$ the DQCMC is indeed able to reproduce the trend that ignition first occurs in the environment of the low scalar 

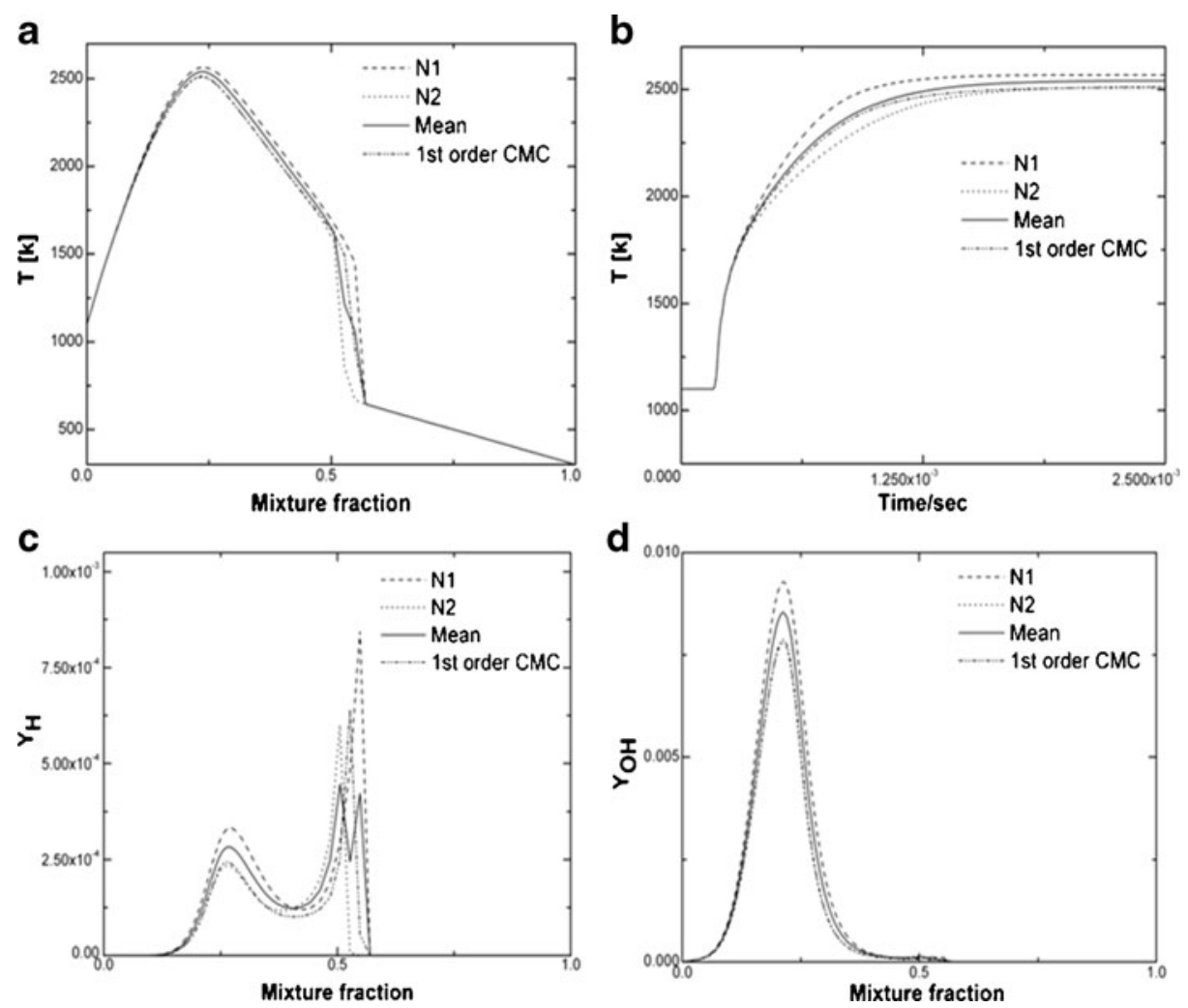

Fig. 5 Conditional temperature as function of mixture fraction (a), maximum temperature as function of time (b), $\mathrm{H}(\mathbf{c})$ and $\mathrm{OH}(\mathbf{d})$ mass fraction as function of mixture fraction at conditions with mean mixture fraction of 0.2

dissipation. The effect of variance on ignition timing that DQCMC hereby captures will be of importance to the results when the DQCMC is coupled to CFD with local variations in scalar dissipation. Finally, Fig. $3 \mathrm{c}$ shows the maximum temperature of the two environments and that of 1 st order $\mathrm{CMC}$ as function of time. It is shown clearly that initially 1 st order CMC ignites earlier than that predicted by DQCMC and has a high maximum temperature, however after ignition environment 2 rapidly takes the lead.

Figure $4 \mathrm{a}$ and $\mathrm{b}$ shows the evolution of the concentrations of some important radicals, $\mathrm{H}$ and $\mathrm{OH}$, in comparison with 1 st order $\mathrm{CMC}$ at times $\mathrm{t} 1$ and $\mathrm{t} 2$. The variation between the conditional mean from the 1st order CMC and the mean from the DQCMC method is small, but noticeable. Moreover it can be seen that initially at time $\mathrm{t} 1$ for both species, environment 2 has higher values than environment 1 . This is due to the enhanced flame development in environment 2. However, at time $\mathrm{t} 3$ environment 1 predicts higher values than environment 2 . This may indicate that although the temperature is higher for lower scalar dissipation, the enhanced chemical reactions lead to that oxidation of such radicals is faster resulting in lower concentrations. This effect should however be investigated further in comparison with direct numerical simulations. 
Finally, the second set of results from conditions close to stoichiometry with mean mixture fraction of 0.2 is shown in Fig. 5. Figure 5a shows the conditional temperature at an instant in time of the two environments of the DQCMC and its mean in comparison with 1st order CMC. The inconsistency between the 1st order CMC and the mean of the DQCMC is shown clearly also in this case, particularly in the ignition zone. Figure $5 \mathrm{~b}$ shows the maximum temperature from DQCMC compared with the 1st order CMC. Similar observation can be noticed in Fig. 5c and d for $\mathrm{H}$ species and $\mathrm{OH}$ species. The local deviations in mixture fraction space are significant, and it shows that the spread around conditional means is non-negligible in advanced turbulent combustion modelling.

It was found that numerically, the DQCMC model was robust and as easy to solve as the 1st order CMC model. The additional terms in the DQCMC-equations did not introduce difficulties regarding convergence of the differential equation solver. Overall, the results show that the DQCMC model has the capability to predict partial extinction and re-ignition and are in a very good agreement with previously observed DNS trend. In the future development of the DQCMC a quantitatively comparison with DNS will be necessary to identify the range for which the simplified assumptions are valid.

\section{Conclusion}

In this work we have presented and validated a method of direct quadrature conditional moment closure (DQCMC) which is based on the direct quadrature method of moment (DQMOM) [12,13]. The analysis is performed for a simple igniting nonpremixed hydrogen flame with a mechanism containing multiple species ( 9 species). The derivation begins with the transport equation using a presumed PDF approach where the joint PDF is expressed as a multi-peak (two environments) delta function; all with a given probability weight, $w_{\alpha}$, representing a distinct mixing-environment. The resulting set of transport equations that need to be solved increases with a factor $N_{e}$, where $N_{e}$ is the number of chosen environments. The final conditional mean for each species is the weighed sum of the contributions from each environment.

Nevertheless the mixing between the environments is properly defined without any need for correction factors. The results have been shown for a special case of two environments with equal weights where the DQCMC has an analytical solution. It is assumed that there is one leading variable in the chemical system such as temperature. The results from the DQCMC model demonstrate to predict qualitatively the trends of partial extinction and re-ignition of the turbulent flames observed from direct numerical simulations. The method can do so with very low additional computational effort. In future work, we will extend the present analysis to different fuels such as hydrocarbon fuels, and will be particularly focusing on the ability of the DQCMC model to capture effects such as soot formation.

Acknowledgements The authors would like to thank Professor E. Mastorakos of Cambridge University for providing the OD CMC code. This work was supported by the UK research council (EPSRC) through the grant EP/F036965/1. T. Løvås would like to acknowledge the support from Churchill College, Cambridge University. 
Open Access This article is distributed under the terms of the Creative Commons Attribution Noncommercial License which permits any noncommercial use, distribution, and reproduction in any medium, provided the original author(s) and source are credited.

\section{References}

1. Peters, N.B.: Turbulent Combustion. Cambridge University Press, Cambridge, UK (2000)

2. Klimenko, A.Y., Bilger, R.W.: Conditional moment closure for turbulent combustion. Progr. Energy Combustion. Sci. 25, 595-687 (1999)

3. Mastorakos, E., Baritaud, T.B., Poinsot, T.J.: Numerical simulations of autoignition in turbulent mixing flows. Combust. Flame 109(1-2), 198-223 (1997)

4. Løvås, T., Lowe, A., Cant, R.S., Mastorakos, E.: Three-dimensional direct numerical simulations of autoignition in turbulent non-premixed flows with simple and complex chemistry. FEDSM2006-98109, ASME Joint US-European FESM (2006)

5. Kronenburg, A.: Double conditioning of reactive scalar transport equations in turbulent nonpremixed flames. Phys. Fluids 16, 2640 (2004)

6. Mastorakos, E., Bilger, R.W.: Second-order conditional moment closure for the autoignition of turbulent flows. Phys. Fluids 10, 1246 (1998)

7. Pitsch, H., Cha, C.M., Fedotov, S.: Flamelet modelling of non-premixed turbulent combustion with local extinction and re-ignition. Combust. Theory Model. 7, 317 (2003)

8. Mitarai, S., Kosály, G., Riley, J.J.: A new Lagrangian flamelet model for local flame extinction and reignition. Combust. Flame 137, 306 (2004)

9. Kronenburg, A., Cleary, M.J.: Multiple mapping conditioning for flames with partial premixing. Combust. Flame 155, 215-231 (2008)

10. Wright, Y.M., de Paola, G., Boulouchos, K., Mastorakos, E.: Simulations of spray autoignition and flame establishment with two-dimensional CMC. Combust. Flame 143, 402-419 (2005)

11. Fox, R.O., Raman, V.: A multienvironment conditional probability density function model for turbulent reacting flows. Phys. Fluids 16(12), 4551-4565 (2004)

12. Fox, R.O.: Computational Models for Turbulent Reacting Flows. Cambridge University Press, Cambridge, UK (2003)

13. Marchisio, D.L., Fox, R.O.: Solution of population balance equations using the direct quadrature method of moments. J. Aerosol Sci. 36, 43-73 (2005)

14. Smith, S.T., Fox, R.O.: A term-by-term direct numerical simulation validation study of the multienvironment conditional probability-density-function model for turbulent reacting flows. Phys. Fluids 19, 085102-1 (2007)

15. Pope, S.B.: Computationally efficient implementation of combustion chemistry using in situ adaptive tabulation. Combust. Theory Model. 1, 41-63 (1997)

16. Tang, Q., Zhao, W., Bockelie, M., Fox, R.O.: Multi-environment probability density function method for modelling turbulent combustion using realistic chemical kinetics. Combust. Theory Model. 11, 889-907 (2007)

17. Brown, P.N., Hindmarsh, A.C.: Reduced storage matrix methods in stiff ODE systems. J. Appl. Math. Comput. 31, 40-91 (1989)

18. Hindmarsh, A.C.: LSODE and LSODI, two new initial value ordinary differential equation solvers. ACM-SIGNUM 15(4), 10-11 (1980)

19. Warnatz, J.: In: Gardiner, W.C. (eds.) Combustion Chemistry. Springer, New York (1984) 